\title{
Cerebral Histoplasmosis in Non-Immunosuppressed Patient - Case Report
}

\section{André Eduardo de Almeida FRANZOI ${ }^{*}$, Nayme Hechem MONFREDINI ${ }^{1}$, Leonora Zozula Blind POPE ${ }^{2}$, Felipe Ibiapina dos REIS and Fabio Antonio TIRONI'}

${ }^{1}$ Medical Student at the University of Joinville Region, Brazil

${ }^{2}$ Pathologist at Hospital Dona Helena and Professor of Pathology at the University of Joinville Region, Brazil

${ }^{3}$ Neurologist at Dona Helena Hospital and Professor of Neurology at Joinville Region University, Brazil

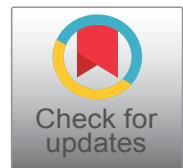

*Corresponding author: André Eduardo Almeida Franzoi, Rua Padre Kolb, 1273 - Postal code 89202-350, Bucarein, Joinville, SC, Brazil, Tel: 55-47-991067944

\begin{abstract}
Histoplasmosis is a disease caused by Histoplasma capsulatum var. capsulatum, which is endemic in Latin America. The manifestation of the disease in the central nervous system (CNS) is more frequent in immunosuppressed individuals with disseminated presentation. Pulmonary manifestations are usually the first symptoms. However, when neurological manifestations are the first clinical manifestations, the diagnosis becomes a challenge. The early diagnosis is fundamental in the final outcome of the patient. The objective is to report a case of difficult identification of cerebral histoplasmosis in a nonimmunosuppressed patient in Joinville, Brazil.
\end{abstract}

\section{Keywords}

Histoplasmosis, Histoplasma capsulatum, Systemic mycosis, Acquired immunodeficiency syndrome

\section{Introduction}

Histoplasmosis is a mycosis caused by dimorphic fungus, $H$. capsulatum. It is considered an endemic mycosis in South America. The patient acquires infection by inhaling microconidia present in nature (like caves with bats). The clinical manifestation ranges from asymptomatic infections to severe disseminated conditions. Severe disease usually affects patients with acquired immunodeficiency syndrome (AIDS) or transplanted patients [1].

The immunodiffusion (ID), complement fixation (CF), and enzyme immunoassay are commonly used for detection of antibodies for diagnosis of histoplasmosis.
Antibodies require 4 to 8 weeks to develop after acute infection and may be negative when a patient is first seen but positive when repeated 1 to 2 months later [2].

Diagnosis can be established by the finding of fungus in organic fluids (such as sputum, blood and cerebrospinal fluid) or tissues. The culture of biological materials is useful to evidences the infection. Treatment of severe, chronic, or localized acute forms may be done with oral azoles (such as itraconazole) and with amphotericin B (preferably lipid formulations). Histoplasmosis represents one of the most important systemic mycoses in the Americas, with wide distribution in Brazil [1].

In this article, we report a case of a male patient, with no underlying comorbidities explaining immunodepression, with negative HIV serology and with a widespread histoplasmosis. The condition was difficult to diagnose as a result of the first signs and symptoms presented by the patient: the central nervous system (CNS) was the most severely affected tissue and the patient's onset of symptoms began.

\section{Case Report}

Patient R. S., male, 55-years-old, metallurgist. Previous comorbidities were systemic arterial hypertension and dyslipidemia. No travel history to another state or contact with caverns and chicken coops.

On the first day of January 2015, the patient was admitted to the hospital unit with horizontal diplopia. From the physical neurological examination, magnetic

Citation: FRANZOI AEA, MONFREDINI NH, POPE LZB, dos REIS FI, TIRONI FA (2018) Cerebral Histoplasmosis in Non-Immunosuppressed Patient - Case Report. Int J Neurol Neurother 5:077. doi. org/10.23937/2378-3001/1410077

Accepted: October 10, 2018: Published: October 12, 2018

Copyright: (c) 2018 FRANZOI AEA, et al. This is an open-access article distributed under the terms of the Creative Commons Attribution License, which permits unrestricted use, distribution, and reproduction in any medium, provided the original author and source are credited. 

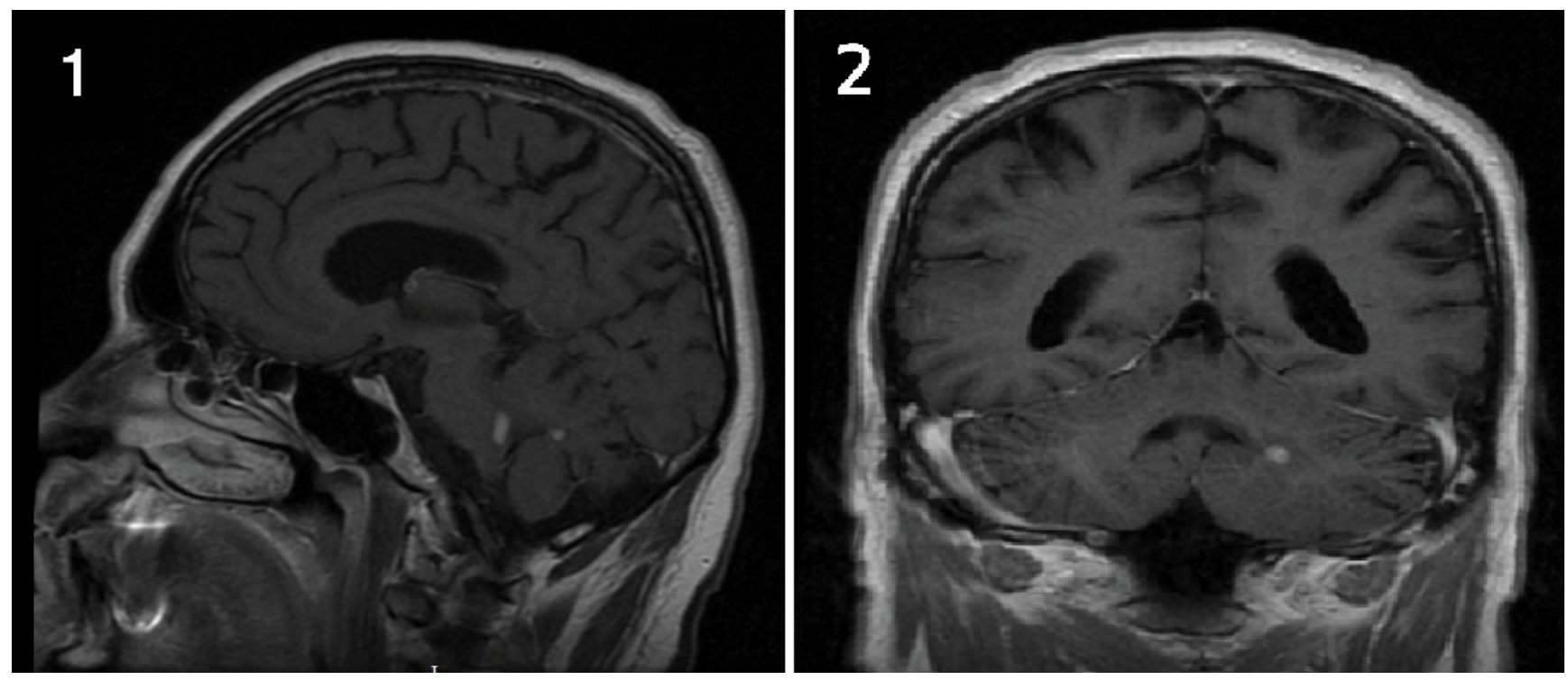

Figure 1: Figure 1 and Figure 2 indicated lesions in the left cerebellar peduncle in sagittal and coronal MRI respectively.

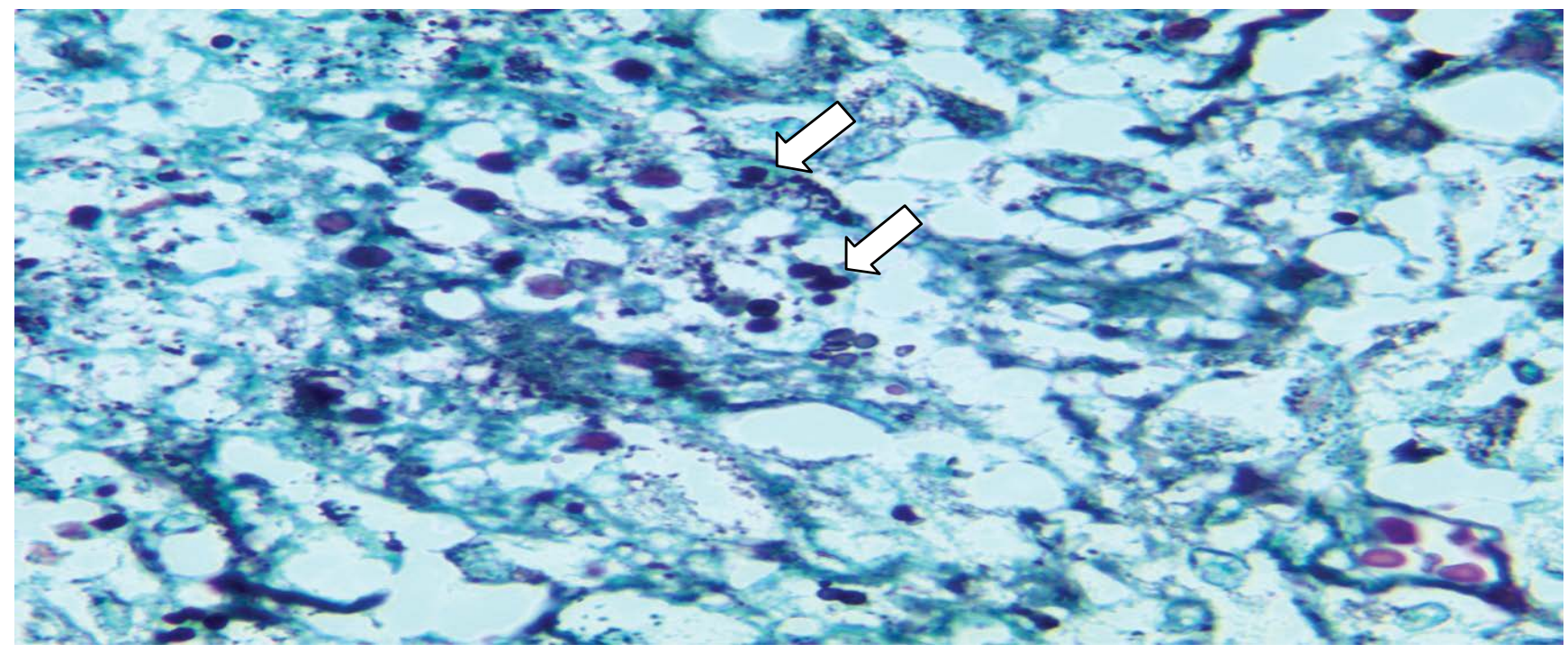

Figure 2: Moderate amount of small, rounded fungal structures, sometimes with single budding within areas of necrosis and giant cells (Grocott staining at 1000x magnification).

resonance image (MRI) was requested. MRI indicated lesions in the left cerebellar peduncle (demonstrated in Figure 1 and Figure 2).

He returned to the hospital 19 days after discharge with severe headache, fever and severe cough, without nuchal rigidity. A new lumbar puncture showed same preview pattern, a chronic meningitis, with 67 cells $/ \mathrm{mm}$ [3], 70\% lymphomononuclear, a low glucose $25 \mathrm{mg} / \mathrm{dL}$ and $168 \mathrm{mg} / \mathrm{dL}$ of protein. Considering neurotuberculosis as the main hypothesis, treatment with Rifampicin $150 \mathrm{mg}$, Isoniazid $75 \mathrm{mg}$, Pyrazinamide $400 \mathrm{mg}$ and Ethambutol $275 \mathrm{mg}$ was started. During treatment, the patient frequently experienced level-of-consciousness oscillation.

The culture of cerebrospinal fluid (CSF) did not identify any pathogenic microorganisms, the patient evolved afebrile, but he was tending to hypotension, mental confusion and spatial disorientation, headache and posi- tional rotational vertigo, vomiting, rotational horizontal nystagmus, in addition to diarrhea. Cerebral biopsy was indicated and revealed only chronic lymphocytic meningitis without granulomas, no viral inclusions, fungal, granuloma, necrotic or neoplastic tissue were found. Biopsy was indicated due to meningeal thickening.

Considering neurosarcoidosis as a possible differential diagnosis, the use of high dose of corticosteroids was started. There was clinical improvement for three days. After one week, the patient had worsened mental confusion and ataxia, a new MRI was performed without new lesions. Fluconazole was suspended, and corticosteroid dose decreased.

After more 5 days, neurological condition worsens with rhomboencephalic signs (bilateral facial palsy, dysarthria and dysphagia, increasing aspiration risk). A new MRI was performed and demonstrated an acute hydrocephalus and ventriculoperitoneal shunt was per- 
formed. One week later, the patient continued worsening his neurological status, with no response even though using tuberculostatics and corticosteroids at moderate doses. The patient was unable to standup and walk without assistance.

On June 15, 2015, the patient kept his status without clinical improvement, and a new cerebral biopsy was indicated and performed. After one week, the anatomopathological examination of the brain biopsy showed a fungus growth result. This exam indicated a probable diagnosis of histoplasmosis (Figures 2, Figure 3 and Figure 4). Then the neurology service restarted amphotericin $B$ (100 mg per day).

In both brain biopsies, the blades were stained by the WADE technique and were negative for BAAR.
After 5 days, there was clinical worsening. A new brain MRI was performed and revealed a collection abscess in the cerebral biopsy area with surrounding contrast enhancement. Local surgical reintervention was required with local drainage and material culture. After one week, there was stabilization of clinical status and the patient was discharged for outpatient follow up.

On August 11, 2015, the patient returned with the same symptoms. A new cerebrospinal fluid test was collected, revealing: glucose $33 \mathrm{mg} / \mathrm{dL}$, protein $304 \mathrm{mg} / \mathrm{dL}$ and leukocytes 50 cells $/ \mathrm{mm}$ [3]. There was stabilization of the clinical status, although there was no improvement in the cerebrospinal fluid test. After one week, the patient was discharged with clinical diagnosis and clinical findings of chronic granulomatous meningitis due to histoplasmosis.

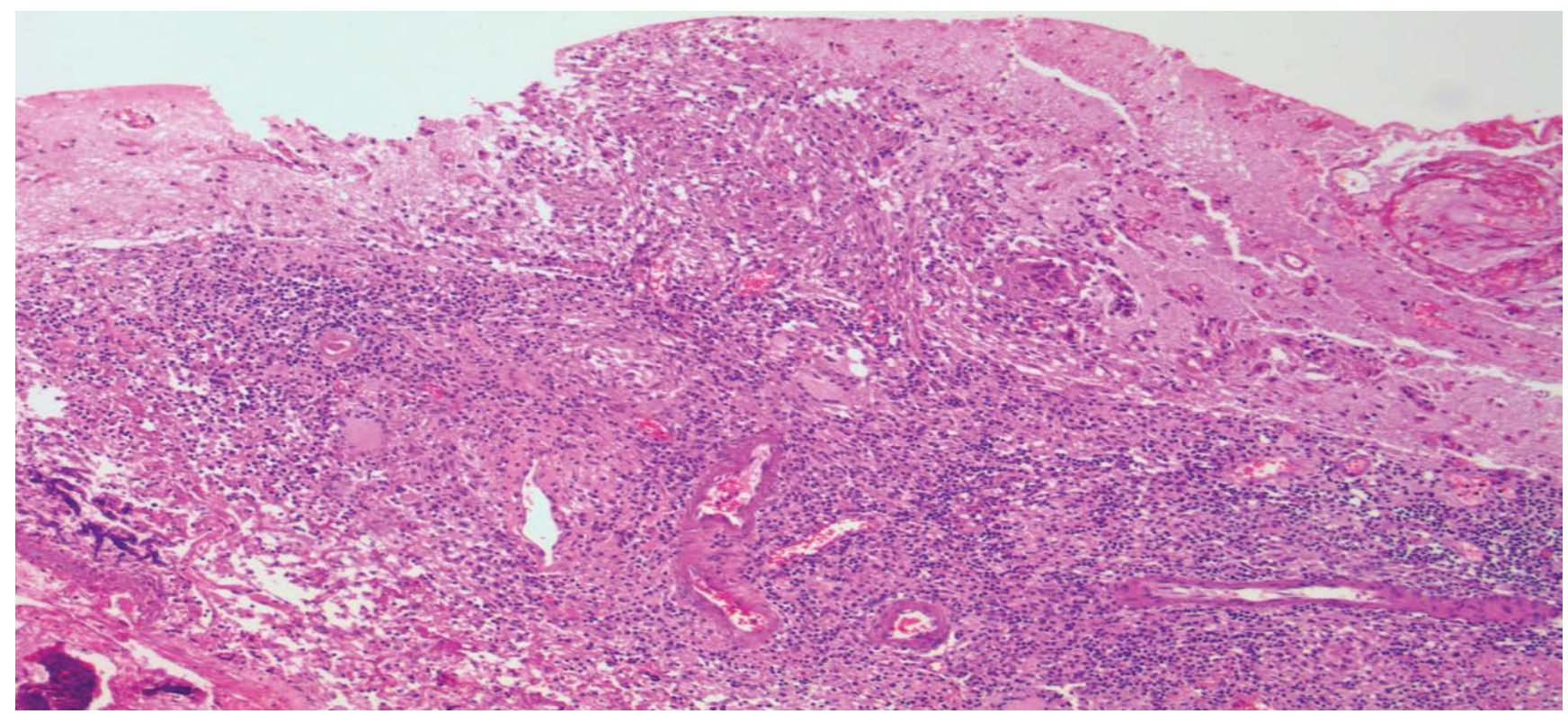

Figure 3: Lymphohistiocytic infiltrate with involvement of the adjacent parenchyma (hematoxylin-eosin increasing by $100 x$ ).

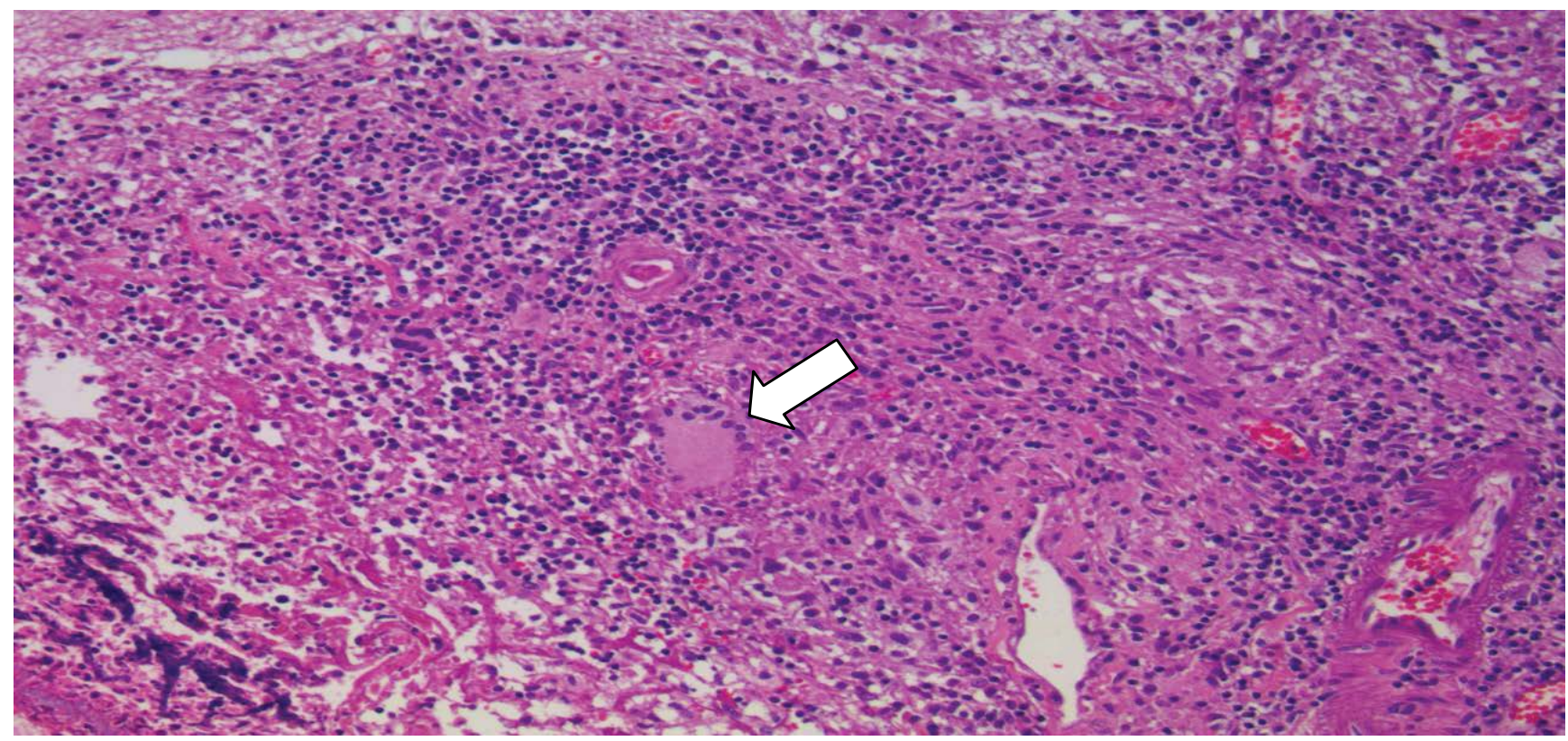

Figure 4: Formation of granulomas and multinucleated giant cells near the areas of necrosis, compromising muscularized wall vessels and cerebral parenchyma (hematoxylin-eosin increasing 200x). 
After more two weeks, the patient completed 75 days of treatment with itraconazole $200 \mathrm{mg}, 8 / 8$ hours. At the outpatient clinic, he had peripheral and central axial ataxia, amyotrophy due to polyradulopathy, hypoacusis, left hemifacial paresis, marked dysarthria and dysphagia.

On the first of October 2015, the plasmatic antigen for persistent histoplasma infection was collected and results positive. Serology for histoplasmosis was performed using the Enzyme-Linked Immunosorbent Assay (ELISA) method. Three collections were carried out: in August, in September and October of 2015. The three serological tests were reagents. The patient was undergoing outpatient follow up and without significant clinical improvement. He was more than 100 days on treatment with itraconazole $200 \mathrm{mg}$.

After five days, the patient returned to the hospital. He was hospitalized with dyspnea, bilateral pulmonary thromboembolism (PET) with right pulmonary opacities. Treatment with enoxaparin $1 \mathrm{mg} / \mathrm{kg} \mathrm{12/12} \mathrm{hours}$ was instituted. After one week, a computed tomography (CT) of the thorax showed pulmonary infarction in the right lower lobe. The specific treatment for pulmonary disease was performed. There was stabilization of clinical status and the patient was discharged for outpatient follow-up again.

On December 28, 2015, the patient returns to the hospital with fever and chest pain. Extensive bilateral PET was diagnosed. The specific treatment was instituted. The CT of the thorax showed localized cavitated lesions occupying the right lung parenchyma, with hydroaeal levels, and may correspond to abscesses associated with loculated empyema. Extensive bilateral pulmonary arterial thrombosis was diagnosed.

Thereafter, the patient presented several episodes of clinical worsening with respiratory insufficiency, dysphagia, pneumonia (with isolation of Pseudomonas aeruginosa and Klebsiella pneumoniae), right side pleural empyema, posterolateral pericardial effusion, pulmonary focus sepsis and shock with orotracheal intubation. The patient's consciousness alternated in periods of mental confusion with drowsiness, also due to encephalopathy secondary to the systemic condition. Intensive bet ineffective support included hydratation, correction of anemia, parenteral nutrition, hydroelectrolytic correction and mechanical ventilation. On April 3, 2016, the patient died, totaling 9 months with itraconazole treatment.

\section{Discussion}

Histoplasmosis is a systemic mycosis caused by a dimorphic fungus, $H$. capsulatum. The habitat of this pathogen is the soil containing feces of birds and bats. Birds do not harbor the fungus due to high body temperature, but bats can be chronic carriers, excreting viable forms in their feces [2].
Histoplasmosis is widely distributed on the South America. In Brazil, prior to the onset of acquired human immunodeficiency syndrome, histoplasmosis was rarely diagnosed and was a curiosity observed only in patients with lymphoma or other neoplasms. In the years 1980 to 1990, with the advent of HIV infection, hundreds of cases of histoplasmosis were observed among patients with the AIDS [3].

The man acquires the infection through inhalation of the conidia present in nature. Most infections are mild or subclinical. Most inhaled conidia remain intact in the pulmonary alveoli. These antigens stimulate an inflammatory response of the host, composed of mononuclear cells [4].

H. capsulatum multiplies within the cells of the monocytic-macrophagic system. From the lungs, they gain the para-hilar and mediastinal lymph nodes. Then they reach the systemic circulation, producing inflammatory foci in other organs such as spleen and bone marrow. After the second week of the onset of infection, a lymphocyte Thelper 1 cellular response is developed. This cell will produce interferon-gamma and other cytokines, which activate macrophages. These cells acquire the ability to lyse the intracellular yeasts of $H$. capsulatum [4].

This response promotes the formation of epithelioid granulomas, with giant cells and caseous necrosis. These factors later give way to fibrosis and calcifications. Specific antibodies are also produced in the patients' serum. However, the antibodies have no protective effect on histoplasmosis. Immunodepression seems to be another important factor in triggering the reactivation of a previous infection [4].

The majority of infections caused by $\mathrm{H}$. capsulatum are subclinical. Symptomatic cases are commonly manifested as self-limited respiratory tract infections. Massive aspiration of conidia of the fungus may lead to the appearance of an acute and severe pulmonary form after an incubation period of one to three weeks. The most common symptomatology consists of fever, chills, headache, myalgias, hyporexia, cough, dyspnea and chest pain [5].

The disease tends to resolve without specific treatment in about two to four weeks, leading to the formation of disseminated calcified nodules in the lungs. Healing of a localized infiltrate may lead to the development of a residual nodule, which progressively increases as fibrotic material is deposited around it. This lesion is called histoplasmoma [5].

Primary infection with $H$. capsulatum can evolve with dissemination of the fungus to the whole organism, particularly to macrophages rich in organs such as liver, spleen, lymph nodes and bone marrow [5].

Rarely a small number of individuals with apparent- 
ly normal immune systems can develop symptomatic disseminated histoplasmosis. In patients with AIDS, histoplasmosis is severe, often fatal and occurs when the TCD4 lymphocyte level is below 200 cells $/ \mathrm{mm}^{3}$. Less than $20 \%$ of them may develop meningoencephalitis with lymphomononuclear pleocytosis and hyperproteinorraquia. These patients report headache, confusion, dizziness, seizures, and paralysis of cranial nerves. The fungus can be visualized and cultured from the CSF, although with low positivity [5].

Some patients with severe disseminated forms may exhibit sepsis-like syndrome with hypotension, shock, disseminated intravascular coagulation, and highly fatal adult respiratory distress syndrome [6].

The diagnosis of histoplasmosis is based on the finding of fungus in secretions and tissues and specific serological reactions. For cases of acute pulmonary histoplasmosis, the history of exposure to potentially contaminated sites is a critical part of the diagnosis. In the absence of this epidemiological data, the serology may confirm this diagnosis, since the fungus finding is exceptional in the respiratory secretions at this stage [5].

Several laboratories prefer to isolate $H$. capsulatum in the mycelial phase and confirm its identification by converting it to the yeast phase after incubation at $37^{\circ} \mathrm{C}$. In progressive disseminated histoplasmosis, the fungus can be seen on microscopic examination in bone marrow smears, peripheral blood, exudate of cutaneous or mucosal lesions, sputum and cerebrospinal fluid [5].

Hemocultures often isolate the causative agent, in particular, in patients with AIDS. In addition, biopsies of skin or mucosa, lung, bone marrow, liver, lymph nodes and intestines demonstrate the presence of sarcoid-like epithelioid granulomas where microorganisms can be seen inside the phagocytic cells. Special colorings, such as Gomori-Grocott, are required for proper visualization of the fungus [5].

At $37{ }^{\circ} \mathrm{C}$, yeasts are small ( 3 to $5 \mu \mathrm{m}$ in diameter), oval and often show single budding. These yeast-like elements may be visualized within macrophages or giant cells and more rarely within the neutrophil polymorphonuclear cells [5].

In the staining of Giemsa or Wright, the fungi show a polar, blue and half-moon color mass. Colored by PAS, they appear in red color. By Grocott silver, in black or dark brown color [5].
Histoplasmosis therapy varies according to the clinical syndrome and host immune status. The fungus is sensitive to drugs such as amphotericin $B$, ketoconazole and itraconazole. Historically, amphotericin $B$ has been the most effective antifungal to treat this mycosis [5].

\section{Conclusions}

The case report demonstrated that rare cases of cerebral histoplasmosis can occur in individuals without immunosuppression. The definitive diagnosis of histoplasmosis in this case was possible only after two cerebral biopsies. Neurological manifestations as the initial presentation are rare and the difficult isolation of the fungus from the brain tissue demonstrates how difficult this diagnosis was.

For the diagnosis of this patient, it was not considered the performance of the western blott serum assay and the achievement of CSF for the realization of PCR methodology. These methods are expensive in Brazil and were not available in the hospital where the patient was treated. This was another topic that challenged the earlier diagnosis of this case.

This case report alerts us about this rare but fatal neurological infection. The health professionals can be able to consider histoplasmosis as a diagnostic hypothesis even in previously healthy patients, as soon as possible, considering that early and correct treatment is the unique way for better outcomes.

\section{References}

1. Wheat LJ, Azar MM, Bahr NC, Spec A, Relich RF, et al. (2016) Histoplasmosis. Infect Dis Clin North Am 30: $207-$ 227.

2. Goodwin Jr RA, Prez RW (1973) Pathogenesis and clinical spectrum of histoplasmosis. South Med J 66: 13-25.

3. Wheat LJ (2001) Laboratory diagnosis of histoplasmosis: update 2000. Semin Respir Infect 16: 131-140.

4. Goodwin RA, Lody JE, Ten Prez RM (1981) Histoplasmosis in normal hosts. Medicine (Baltimore) 60: 231-266.

5. Wheat LJ, Freifeld AG, Kleiman MB, Baddley JW, McKinsey DS, et al. (2007) Clinical practice guidelines for the management of patients with histoplasmosis 2007 update by the Infections Diseases Society of America. Clin Infect Dis 45: 807-825.

6. Wheat LJ, Musial CE, Jenny-Avital E (2005) Diagnosis and management of central nervous system histoplasmosis. Clinical Infectious Diseases 40: 844-852. 